\section{$\underset{\substack{\text { hommes } \\ \text { \& migrations }}}{ }$}

\section{Hommes \& migrations}

Revue française de référence sur les dynamiques

migratoires

$1301 \mid 2013$

Migrations et mondes ruraux

\title{
Le stradivarius et la frite
}

\section{Mustapha Harzoune}

\section{OpenEdition \\ Journals}

\section{Édition électronique}

URL : http://journals.openedition.org/hommesmigrations/1957

DOI : 10.4000/hommesmigrations. 1957

ISSN : 2262-3353

\section{Éditeur}

Musée national de l'histoire de l'immigration

\section{Édition imprimée}

Date de publication : 1 janvier 2013

Pagination : 194-197

ISBN : 978-2-919040-21-6

ISSN : $1142-852 X$

\section{Référence électronique}

Mustapha Harzoune, «Le stradivarius et la frite », Hommes \& migrations [En ligne], 1301 | 2013, mis en ligne le 29 mai 2013, consulté le 22 septembre 2020. URL : http://journals.openedition.org/ hommesmigrations/1957 ; DOI : https://doi.org/10.4000/hommesmigrations.1957

Ce document a été généré automatiquement le 22 septembre 2020.

Tous droits réservés 


\title{
Le stradivarius et la frite
}

\author{
Mustapha Harzoune
}

1 Connaissez-vous le secret des stradivarius? La question peut paraittre saugrenue dans une revue spécialisée en sociologie des migrations. Et pourtant. Michaël Ferrier déjà, avec le phénomène dit du "fantôme", fila la métaphore musicale pour dépoussiérer le récit national (lire Sympathie pour le fantôme, Gallimard, 2010, prix littéraire de la Portée Dorée, 2011). C'est le Dépêches notes du 4 décembre 2012 de France Musique qui, citant le Daily Telegraph du 2 décembre, donne à réfléchir. Le "mystère" du "son magnifique" des stradivarius viendrait de leurs "imperfections". "Le docteur Franco Zanini de l'université de Trieste, en Italie, a passé les divins violons au microscope et a montré que les multiples retouches, les asymétries, des petites taches, des micro-fissures sont la clef du mystère: c'est parce que ces instruments ne sont pas parfaits qu'ils développent des harmonies, des sonorités uniques."

2 Cela rappelle les "charmes de la souillure" exposés par Marcel Detienne dans Comment être autochtone. Du pur Athénien au Français raciné (Seuil, 2003). Les imperfections, les ajouts, les retouches, l'impur, ne réservent-ils pas de mystérieuses réussites? Certes, rien ne va de soi. Il ne s'agit pas d'une grâce tombée du ciel, d'un mode d'emploi, le petit nécessaire de l'apprenti sorcier qu'il suffirait d'ouvrir. Il faut plutôt s'aventurer dans les méandres d'un processus fait de tâtonnements, d'une dose de bon sens, d'impasses et de souffrances. Du point de vue des migrations, de la rencontre des peuples et des cultures, il y entre la nécessité de l'adaptation, l'acceptation des "fissures", des " imperfections" et donc la fragilité des constructions identitaires. Cette disponibilité aux changements présuppose non pas une conscience des différences mais des "écarts" dont chacun peut se nourrir, se régénérer ou se conforter.

\section{La dynamique de l'écart}

Le Monde du 2 janvier 2013 se demande si "la frite est belge ou française". De cela, Roel Jacobs, spécialiste de la culture bruxelloise, n'a que faire: "À ses yeux, le sujet le plus intéressant réside dans la manière dont ce plat a été 'adopté' des deux côtés de la frontière. En France, il se décline généralement comme une garniture, en accompagnement d'une viande, alors 
qu'en Belgique, il se déguste le plus souvent seul. 'Nous, les Belges, avons fait de la frite un produit noble, pas un simple légume, estime Albert Verdeyen, cuisinier et coauteur de l'ouvrage Carrément frites [...]'"

4 La notion d'écart montre comment l'humanité développe, dans sa diversité, mille et une variations et potentialités. Chaque peuple, chaque culture met "à sa sauce" tel ou tel aliment, telle ou telle pratique, hiérarchise telle ou telle valeur, telle ou telle règle. “ Ainsi, en France, le bâtonnet de pomme de terre se mange-t-il piqué sur une fourchette et dans une assiette, à l'heure des repas, alors que chez nos voisins, il est consommé avec les doigts dans un cornet et à n'importe quelle heure. Sur les places, le long des boulevards ou devant les gares de Wallonie comme de Flandre a dès lors fleuri un réseau serré de fritkoten, ou baraques à frites."

5 De cet infime écart culturel quant à l'usage d'un bâtonnet de pomme de terre frit dans un bain d'huile peut naître une large amplitude sociologique : "À l'intérieur des baraques, les choses changent : les frituriers d'origine étrangère sont de plus en plus nombreux, comme en témoigne l'entrée du mot 'frietchinees' ('friturier chinois') dans le dictionnaire de référence de la langue néerlandaise en Belgique." "Les Chinois ne sont toutefois pas les seuls nouveaux arrivants dans le secteur, puisqu'ils ne représentent que $1 \%$ des propriétaires des quelque 5000 fritkoten du royaume, relève le directeur du Musée de la frite de Bruges, Eddy Van Belle. 'Friturier turc' ou 'friturier marocain' méritaient plus de figurer dans le dictionnaire, selon le spécialiste" (bigbrowser.blog.lemonde.fr du 19 décembre). Allez savoir si, derrière ces "fissures" et "retouches", ne se prépare pas une mystérieuse création à l'instar de ce sandwich vietnamien ou "bánh mi" qui mêle savamment cuisines française et vietnamienne, legs colonial et globalisation, immigration et adaptation. La préparation, goûteuse et peu coûteuse, prospère dans certains quartiers de Paris. Sans doute une “ imperfection" au regard des gardiens de l'ordre de la baguette-jambon-beurre ou de la traditionnelle soupe phô, mais, à n'en pas douter, une trouvaille qui, si elle ne réserve pas pour le palais les subtilités d'un stradivarius pour les oreilles, témoigne du génie de l'Homo migrator et des délicatesses de la vie.

\section{E pluribus unum}

6 "L'Homo parisianus" soi-même barbote aujourd'hui dans le nouveau (Le Monde, 16 novembre). Exit le titi d'antan, la gouaille du prolo et la contestation du pavé... Les 2,2 millions d'habitants de la "ville-monde" se partagent en "deux mouvements" opposés. "D'une part, la gentrification, liée à la surreprésentation des catégories socio-professionnelles supérieures et à la hausse du foncier. De l'autre, la montée en puissance de l'immigration. [...] Comme toutes les grandes métropoles, le Paris de 2012 est donc embourgeoisé et cosmopolite."

7 "Selon l'Insee, $38 \%$ des habitants de la capitale âgés de 18 à 50 ans étaient immigrés (20\%) ou enfants d'immigrés (18\%) en 2007. Et la part de la population étrangère est plus importante à Paris (15\%) que dans d'autres grandes villes. [...] Grâce à un marché du travail dynamique, y compris dans des secteurs peu qualifiés, la capitale demeure attractive pour les étrangers et les scores du FN y sont toujours bien en dessous de la moyenne nationale."

8 Le quotidien note qu'"une petite bourgeoisie d'origine africaine, notamment maghrébine, émerge. S'il n'investit pas encore les quartiers les plus prisés de la capitale, le beurgeois parisien évolue sans heurts dans une ville multiculturelle où les valeurs progressistes sont plus ancrées qu'ailleurs. Individualiste, il associe souvent un mode de vie urbain à la pratique d'un islam 
culturel qui fait de lui une cible marketing: dans les supérettes parisiennes, plats cuisinés, pizzas et foie gras halal lui sont destinés."

Reste à harmoniser cette diversité, à "maintenir le lien entre des catégories de plus en plus hétérogènes". La fonction en incomberait à "une classe moyenne administrative, grâce à laquelle la capitale fonctionne". Faut-il ajouter cette réflexion de l'Algérien Mohamed Dib : "Un migrant qui ne reste qu'un immigré est un migrant raté"?

10 L'exposition de l'IMA consacrée aux Mille et Une Nuits contribue aussi à bousculer les fantasmes de perfection et d'unicité et rappelle que les intégristes, tous les intégristes, malades de la pureté, haïssent la culture et les lieux de mémoire. Pour Malek Chebel, Les Mille et Une Nuits sont "le plus formidable manifeste anti salafiste jamais écrit": "Nous assistons depuis trente ans, dans tout le monde musulman, et désormais dans l'élite européenne, à un gigantesque refoulement de la grande culture arabe classique et populaire, érotique aussi, une véritable démolition d'un passé brillant [...]. Selon lui, les puristes et les piétistes actuels de l'islam, les salafistes et la plupart des Frères musulmans, veulent revenir à la religion $d u I^{e}$ siècle - une véritable "régression intellectuelle", qui, en retour, alimente la virulence des Européens contre tous les musulmans" (sexe.blog.lemonde.fr, 30 décembre).

11 La virulence et la perte de repères. Ainsi, Stéphanie Le Bars sur son blog, le 6 décembre, rappelle: "À Montargis, la directrice d'une école maternelle avait décidé de supprimer la traditionnelle visite du Père Noël [...] 'afin de respecter les différentes croyances ainsi que les valeurs de l'école laïque'. [...] Au Havre, cette fois, ce sont des milliers de crèmes au chocolat, contenant de la gélatine de porc, qui jeudi 29 novembre ont été détruites [...]. 'Des employés [non musulmans] des cantines de la ville nous ont fait part de leur inquiétude au cas où l'information selon laquelle les desserts du jour contenaient de la gélatine de porc arriverait aux oreilles des enfants', explique, Philippe Brunel, le directeur général adjoint au développement social et à la famille, à la mairie."

12 Pour Dounia Bouzar, "cela ne devrait pas être compliqué d'expliquer à toutes les familles que le Père Noël, ce n'est pas la même chose que la crèche, que cette fête est passée dans le registre culturel et qu'elle n'est pas discriminante. Il faut par ailleurs assumer le fait que les normes françaises, le dimanche férié ou les vacances de Noël, sont issues d'une tradition chrétienne. Quitte à ce qu'un jour des fêtes venues de minorités religieuses basculent aussi dans du culturel partagé par tous". Quelques "retouches" et "fissures" en somme.

Pour Malek Chebel, "la beauté est une arme contre l'oubli et le déni de soi. Elle permet aux jeunes Français issus de l'immigration de se connecter à une histoire plus flamboyante. L'oubli des chefs-d'œuvre de sa propre culture, encouragé par des intégristes bornés [ou 'ces vieux barbons' de l'UOIF, dixit Chebel] [...], contribue à isoler les populations immigrées dans leur cage d'escalier, y compris celles qui sont nées en France".

Le stradivarius, la frite, Paris, l'islam, le vivre ensemble... seraient donc perfectibles, soumis, pour le meilleur et non pour le pire, au travail de la "souillure" et des "retouches ". La France aussi est "une invention", comme le titre l'entretien de Julie Clarini avec les historiens Joël Cornette et Johann Chapoutot, le premier responsable de l'édition chez Belin de treize tomes illustrés d'une Histoire de France, le second d'une Histoire de la France contemporaine au Seuil (Le Monde, 19 octobre).

Pour Joël Cornette, "l'histoire de France est elle-même une histoire connectée, dès l'origine. Après tout, les Francs ne sont pas français! L'histoire que nous proposons n'est pas une histoire enfermée dans les frontières hexagonales, elle est ouverte aux quatre vents". Johann Chapoutot note que "ces dernières années, les historiens ont [...] beaucoup parlé de perspectives 
'transnationales' (les circulations, les effets de retour, les échanges...), mais le 'transnational' présuppose, par définition, la nation comme premier élément de l'échelle; du reste, elle a été et persiste à être un fait majeur et structurant du monde contemporain [...]". Pour lui, "il est important de ne pas laisser la nation à ceux qui l'envisagent uniquement dans une perspective obsidionale, comme une citadelle assiégée sur laquelle des hordes d'étrangers viendraient se jeter".

Joël Cornette ajoute "que ces discours-là sont d'autant plus absurdes que la singularité de la France est précisément qu'au moment où elle se constitue comme Nation, au moment de la Révolution, elle nourrit des idéaux transnationaux (liberté, égalité, fraternité). Ainsi, en un sens, elle est une nation internationale dès l'origine. Par ailleurs, elle se nourrit des apports étrangers. [...] Aucune période de l'histoire de France ne porte trace de la pureté que certains voudraient aujourd'hui revendiquer".

\section{Couvrir le tintamarre des boutiquiers}

17 Et pourtant, "les préjugés, qui étaient en recul depuis 2005, sont repartis à la hausse depuis deux ans. La crise économique n'est pas seule en cause. 'Lorsque le politique se permet de raisonner en généralités sur tel ou tel groupe ethnique, cela contamine le débat public et favorise l'expression des préjugés', constate Jérôme Sainte-Marie, directeur du pôle Opinion de l'institut de sondage CSA [...]" (Le Monde, 9 novembre). "Depuis 2010 et le discours de Grenoble de Nicolas Sarkozy sur l'immigration, les Français se montrent de plus en plus intolérants. La campagne présidentielle de 2012, marquée par une 'ethnicisation' du discours politique, s'inscrit dans le même registre. 'Jamais, durant la Ve République, une élection n'a été autant entachée par des propos racistes ou xénophobes, venant non seulement du $F N$ mais aussi d'une partie de la droite, ce qui a fait sauter les verrous', souligne Sipa Réda Didi, délégué général de Graines de France", un cercle de réflexion sur les quartiers populaires.

Pour bousculer ces préjugés et réintroduire un peu de complexité, la presse multiplie les articles et les dossiers. L'Express.fr (8 novembre) reprend une étude de l'Observatoire des inégalités et "démontre que les enfants d'immigrés réussissent mieux à l'école que les enfants français". L'Expansion-L'Express.fr (14 novembre) montre que les chefs d'entreprise reconnaissent mezza voce que la main-d'œuvre immigrée est “ indispensable dans plusieurs secteurs d'activité": "Selon un document de travail du Centre d'analyse stratégique intitulé 'L'emploi et les métiers des immigrés', ceux-ci représentent plus de $30 \%$ des effectifs dans les métiers du nettoyage, des employés de l'hôtellerie, et entre 20 et $30 \%$ pour les secteurs de la sécurité, du bâtiment et des travaux publics, de la restauration... Les informaticiens étrangers sont également très prisés."

"Dans les métiers de la sécurité, nous avons 40000 employeurs qui cherchent des salariés. Alors oui, les travailleurs étrangers permettent aux entreprises de tourner", confirme Michel Ferrero, président du Syndicat national des entreprises de sécurité, et Cécile Jolly, économiste au Centre d'analyse stratégique, réitère qu'"entre autochtones et immigrés", "il y a peu de concurrence, car les uns et les autres n'évoluent pas tout à fait sur les mêmes marchés du travail".

20 Schizophrénie médiatique oblige, la couverture de L'Express du 14 novembre illustre son dossier sur les "coûts" de l'immigration par une femme en burqa entrant dans une agence de Pôle emploi. Pourtant, à l'intérieur du magazine, Christophe Barbier écrit : “ L'immigration rapporte plus à la France qu'elle ne lui coûte. Économiquement et socialement, 
elle est une 'bonne affaire' à court, moyen et long termes. À court terme, parce que les immigrés occupent des postes dont les Français ne veulent pas, cotisent et consomment. À moyen terme, parce que les étudiants étrangers accueillis - et les rejeter, comme le fit la droite, était une faute - sont un excellent investissement pour le futur rayonnement international de la France. À long terme, parce que le dynamisme démographique du pays, dû en partie aux immigrés, assurera notre puissance en Europe, notamment face à l'Allemagne."

Le dossier reprend les données des travaux de Xavier Chojnicki et Lionel Ragot (voir Kiosque $n^{\circ}$ 1297). Les deux chercheurs reviennent sur les chiffres de Jean-Paul Gourévitch qui évaluait en 2009 le coût de l'immigration à quelque 37 milliards d'euros de déficit annuel. La différence entre les deux études "réside dans la définition de la population immigrée retenue. Nous avons choisi les critères de l'Insee: être né étranger dans un pays étranger [...]. Jean-Paul Gourévitch ajoute [...] 2 millions de personnes: les enfants d'immigrés [...], mais le problème réside dans le fait qu'il ne les comptabilise que lorsqu'ils sont mineurs et qu'ils pèsent le plus sur les dépenses publiques - via l'éducation notamment - et pas quand ils sont majeurs et contribuent, en payant des cotisations et des impôts, à la collectivité." Par ailleurs, "cette étude impute aux immigrés le coût de la contrefaçon (2,2 milliards d'euros), celui de la prostitution (1,4 milliard d'euros) ou de l'aide publique au développement [...]. Tout cela est un peu surprenant. Dernier point, nous estimons qu'elle surévalue les dépenses de santé des immigrés [...]."

Jean-Paul Gourévitch, lui, a repris sa calculette, réévalué justement les "recettes provenant des enfants d'immigrés" (site du Cri du contribuable, 16 novembre), élargi sa recherche aux collectivités et territoires d'outre-mer et "à l'ensemble des populations concernées (migrants réguliers, irréguliers et descendants directs de migrants)". Résultat, le déficit tombe en l'espace de quatre ans de 37 milliards annuels à 17,4 milliards. Cela n'empêche pas, le 20 novembre, le très responsable site du contribuable d'écrire : " $L a$ question de réduire le coût de l'immigration doit se poser sans tabou" (et sans erreurs).

Laissons là le charivari statistique pour méditer, stradivarius à l'oreille et frites en bouche, sur "les charmes de la souillure" et des imperfections, les pétulances de l'impureté et de l'inachevé. Peut-être serait-ce la meilleure façon de laisser monter de nouvelles et harmonieuses "sonorités" et de couvrir le tintamarre des zélotes de la pureté et des boutiquiers.

\section{AUTEUR}

\section{MUSTAPHA HARZOUNE}

Journaliste 